\title{
Response enhancement in children as a function of blocking at different distances from start and goal*
}

\author{
THOMAS CLIFFORD and MICHAEL S. CROSS \\ Kenyon College, Gambier, Ohio 43022
}

Fourteen second- and third-grade boys played a game in which they attempted to fill a tube to various levels with marbles. E blocked their progress at numerous points in an attempt to assess the contributions of distance from the start, distance from the goal, and total game length on S's response of hitting a dowel following blocking. Results suggested that distance from the start is a more potent variable affecting response strength following blocking than either distance from the goal or total game length.

Haner \& Brown (1955) had children play a game in which they attempted to fill all the holes of a 36-hole board with marbles. On various trials $S$ was blocked at five distances from the goal $(0,4,9,18$, and 27 marbles). On such trials a buzzer sounded as the marbles fell through the holes and out of view of $S$, who then had to depress a plunger to stop the buzzer before he could begin a new trial. Haner and Brown interpreted their results as showing that the extent of depression of the plunger, or "instigation to action," was directly related to goal proximity at the time of blocking.

Since Haner and Brown held total game length constant, an equally logical interpretation of their results would be that the extent of depression was related to distance from the start, or effort expended. There is more recent evidence from studies with rats to support this alternative. A report by Amsel, Ernhart, \& Galbrecht (1961) suggests that a longer run to failure of an expected reward results in a greater frustration enhancement effect, and Lewis (1964) presented results relating incentive value of a reward to the amount of effort required to obtain it.

The present study was designed to explore the relative contributions of the three variables confounded in the Haner and Brown study-goal proximity, distance from start, and total game length-to response strength following blocking of a goal-directed act.

\section{SUBJECTS}

The Ss were 14 boys selected at random from the second and third grades of a public elementary school in Gambier, Ohio.

\footnotetext{
*This research was supported by National Institute of Mental Health Grant NM 16762-01. The cooperation of Dr. Edward A. Greenwald, Principal of the Meadow Lane School of Gambier, Ohio, and of Dr. Earl E. Hogan, Superintendent of Schools, Mount Vernon City Schools, Mount Vernon, Ohio, is gratefully acknowledged.
}

\section{APPARATUS}

The game board was an upright, light blue, 3/8-in. plywood board, 24 in. square, upon which was mounted a 3/4-in. aluminum tube, 24 in. long, cut open on the face side so that marbles within could be seen and set at an angle of 30 deg from left to right across the board. Red 6-V lights were mounted above the tube and parallel with it to define games of different lengths. A hand-operated $110-\mathrm{V}$ solenoid was mounted at the lower end of the tube so that its core blocked the tube when the circuit was open. When the switch was activated, the core was retracted, and marbles in the tube rolled down a chute through a hole in the board out of sight of S. The solenoid, chute, and hole were shielded with red cloth. A brightly painted smiling clown's face, made of plywood and raised about $1 \mathrm{in}$. above the game board, was located in the upper center of the board. The clown's nose was a $7 \frac{1}{2}-\mathrm{W}$ red light which could be flashed off and on by E with a pushbutton switch. Occurring simultaneously with the flashing nose was the sound of a muffled door bell, which was mounted on the back of the game board. A large bowl of marbles was placed in front of the board.

A second part of the apparatus consisted of a red wood box, $91 / 2$ in. square and $6 \frac{1}{2}$ in. high, with a frame above to support a $1 \frac{1}{2}$-in. dowel with a padded top in a vertical position. The dowel rested on 4 in. of foam rubber inside the box. Graph paper ruled in millimeters was taped around the dowel. A plastic ring was cut to fit snugly around the dowel, and a lower support prevented the ring from moving down when the dowel was depressed. When the dowel was depressed and then bounced back, the ring adhered to the dowel and thus gave a reading in millimeters of the extent of downward displacement of the dowel. Displacement by multiples of a standard weight revealed a linear scale. Also fastened to the box was a $6 . \mathrm{V}$ buzzer which was activated simultaneously with the solenoid of the game board. The buzzer, plastic ring, and graph paper were concealed from $\mathrm{S}$ by a plywood shield which bore a second clown's face with a "down-at-the-mouth" expression and coloring markedly different from the clown of the game board.

\section{PROCEDURE}

Each $\mathrm{S}$ was escorted from the classroom by $E$ to a small private room in the school and instructed as follows:

"You stand here. (S was positioned facing the game board with the dowel immediately to his right.) We are going to play a game with these two clowns. This is the happy clown. (E pointed to the game board.) And this is another clown. (E pointed to the dowel apparatus.) What you are supposed to do is take marbles from the bowl one at a time and put them in the tube, like this. (E demonstrated.) Now you try it. (S inserted a marble.) That's fine.

"To win a game you have to pile the marbles up in the tube as high as a light. This light would be a short game; this would be a long game. (Here E lighted first the 4- and then the 32-length game lights placed above the tube.) The happy clown wants you to win the game. When you pile the marbles up as high as a light and win, he is happy and his nose lights up, like this. (E flashed the nose.) Every time you make his nose light up by winning a game, he gives you a prize. You will get the prizes later when all the games are over.

"But this other clown wants you to lose the game. Sometimes he makes a noise, and all the marbles fall out of the tube. When he makes the noise, you lose the game. You can stop the noise by hitting 'this.' (E hit the dowel down with his fist.) Now you try it. (S hit the dowel.) When the clown makes the noise and you lose the game, you have to hit 'this' and stop the noise before you can start a new game and try to win another prize.

"Let's try the last part. Show me what you will do when the clown makes a noise and the marbles fall out. (E activated the solenoid and buzzer; S hit the dowel; E stopped the buzzer.) That's right. You want to make the happy clown's nose light up and win a prize, but if this clown makes a noise, you have to hit 'this.' Do you understand how to play the game? Good. You do not have to hurry; you can take your time playing the game. Watch for a light to come on over the tube, and when one does, you can start putting the marbles in the tube. Let's play." E then took a seat behind the game board and began the trials. E lighted the appropriate game-length light, and $\mathrm{S}$ began to insert the marbles. On blocked trials $E$ activated the solenoid and buzzer after the appropriate number of marbles had been inserted, recorded the extent of depression of the 
Table 1

Distances from Start and Distances from Goal for Games Lost. with Total Game Lengths in Cells Representing Games Played

\begin{tabular}{|c|c|c|c|c|c|c|c|c|}
\hline & & \multicolumn{7}{|c|}{ Distance from Goal } \\
\hline & & 2 & 4 & 6 & $\mathrm{~s}$ & 12 & 16 & 24 \\
\hline & 2 & 4 & & 8 & 10 & 14 & & 26 \\
\hline & 4 & & 8 & & & 16 & & \\
\hline Distance & 6 & 8 & & & 14 & & & \\
\hline from & 8 & 10 & & 14 & 16 & 20 & & 32 \\
\hline Start & 12 & 14 & 16 & & 20 & 24 & & \\
\hline & 16 & & & & & & 32 & \\
\hline & 24 & 26 & & & 32 & & & \\
\hline
\end{tabular}

Table 2

Vean Dowel Displacement in Millimeter for Game: Lost

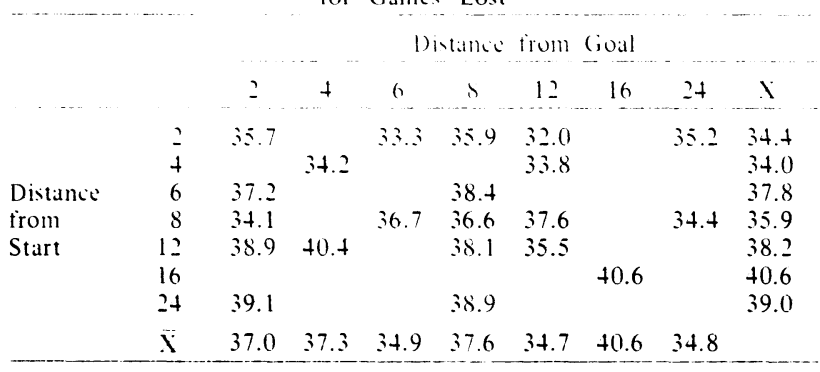

dowel, and reset the plastic ring. On win trials $E$ flashed the nose and rang the bell and then emptied the marbles from the tube for the next trial. At the close of the experimental session. $S$ received a cup of M\&Ms as prizes.

An experimental session consisted of 30 trials, of which Trials 1. 4, 9, 15, 19. 24 . and 27 were wins, Trials 2 and 3 were warm-up losses, and the remaining trials were recorded losses. The recorded losses were randomly ordered without replacement for each $\mathrm{S}$ from the filled cells of the matrix shown in Table 1 . The seven wins and two warm-up losses were also ordered by separate random selection without replacement from the same cells. An added constraint on the total order was that each series of four games include at least one game from each length class. length classes being defined as "short" (4 8 , and 10 marbles), "medium" $(14,16$, and 20 marbles), and "long" $(24,26$, and 32 marbles).

\section{RESULTS AND DISCUSSION}

Each $S$ contributed one dowel displacement score in millimeters for each of the 21 different games on which blocking occurred. An analysis of variance of order effects showed no significant effects related to game order, $\mathrm{F}(20,260)<1$. Mean displacement scores

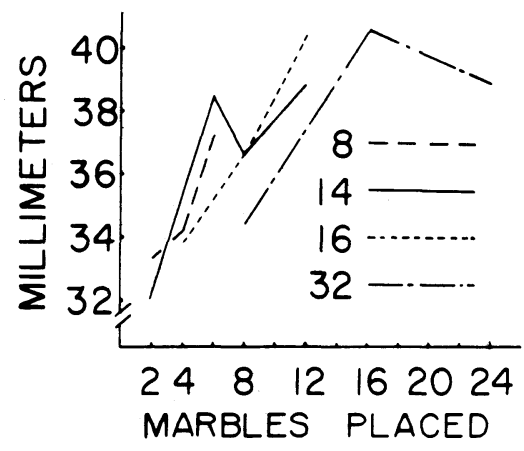

Fig. 1. Response strength as millimeters of displacement as a function of number of marbles placed prior to blocking: parameter is total game length. for each game and for each blocking point are shown in Table 2.

The design included four sets of games in which total length was constant and in which blocking occurred at different distances from the start. or at different distances from the goal. These were sets which. like the Haner and Brown set, confounded start and goal distances. The total game length and number of marbles placed prior to blocking for these sets were as follows: Length $8(2,4$, and 6 marbles before blocking): Length 14 (2, 6. 8, and 12 marbles before blocking); Length 16 (4. 8 , and 12 marbles before blocking): and Length 32 ( 8,16 , and 24 marbles before blocking). Figure 1 shows the amount of displacement as a function of number of marbles placed for each of these sets: in general. response strength increased as $\mathrm{S}$ was blocked closer to the goal. or farther from the start. Analyses of variance for each set revealed marginally significant effects for the games of Length 8 . $F(2,26)=2.58, \quad p<.10$. and 14 . $\mathrm{F}(3,39)=2.49, \quad \mathrm{p}<.10$. and significant effects for the games of Length 16. $F(2,26)=5.60, p<.01$. and 32 . $F(2,26)=5.00, p<.05$. These findings corroborate the results of Haner \& Brown (1955)

Effects of distance from the goal. or goal proximity, were most readily assessed by examining the trend in the marginal bottom row of Table 2. showing averages down columns, each column representing a distinct distance from the goal. Although an analysis of variance of these scores showed a significant $F$-ratio for different distances from the goal, $F(6.78)=3.22$, $\mathrm{p}<.01$, examination of Table 2 reveals no plausible systematic trend in the series.

Effects of distance from start were also most readily assessed by analyzing averages across rows of Table 2, i.e.. those averages shown in the last column. Analysis of variance of these data were also significant. $F(6,78)=8.06, p<.001$, and examination of the trend down the marginal column suggests that response strength ir sreased directly with distance from the start.

Analyses based on fewer cells drawn from within the matrix of Table 2 revealed generally less reliable relations. Several matrices were drawn from the set of games tested where distance from goal could be analyzed as an orthogonal dimension with respect to distance from start. For example. each row was analyzed separately, giving a constant distance from start for each row with distance from goal varying across the row. Examination of the rows of Table 2 offer little suggestion of systematic trends related to distance to goal, and analyses of variance of each row revealed only one of six which exceeded the level of marginal significance, that for distance from start held constant at 12 , $F(3.39)=2.32 . p<.10$.

Similar analyses down columns. involving variation in distance from start with distance from goal held constant. showed significant or marginally significant effects for but two of the six columns. those two where distance from goal was constant at $+. F(1.13)=9.92 . p<.01$. and 12. $F(3.39)=2.20, p<.10$. Both of these columns suggest a direct relation between response strength and distance from start.

Four matrices within Table 2 involved multiple points on both dimensions. distance from start and distance from goal. respectivels: (1) a 2 by 2 matrix with 4.12 and 4.12. $F(1.13)=7.20, p<.05$ for distance from start and $F(1.13)=6.77$. $p<.05$ for distance from goal. with the former reflecting increasing response strength and the latter decreasing response strength, or increasing response strength with increasing goal proximity: (2) a 2 by 4 matrix with 2.8 and 2. 8. 12. 24. $F(1.13)<1$ for distance from start and $F(3.39)<1$ for distance from goal: (3) a 4 by 2 matrix with 2.8.12.24 and 2. 8 . $F(3.39)=2.71 . p<.10$ for distance from start and $F(1.13)<1$ for distance from goal. the former reflecting a direct relation between response strength and distance from start: and (4) a 3 by 3 matrix with 2 . 8.12 and $2,8,12, F(2,26)=2.10 . p>.10$ for distance from start and $F(2,26)=1.04$. $p>.10$ for distance from goal. There were no significant Start by Goal interaction effects. As in the analyses of marginal 
averages of Table 2, analyses of matrices drawn from the within-cells suggest more reliable systematic relations for distance from start than for distance from goal, but the within-cells analyses were less stable than analyses of marginal averages.

The games tested were also analyzed in terms of total game length and proportional distances from start and goal. An analysis of averages of the nine different game lengths, derivable from Table 2, showed no reliable differences related to total game length, $\mathrm{F}(8,104)=1.20, \mathrm{p}>.10$. Also derivable from Table 2 was a set of games where total length varied and proportional distance from start and goal was constant at $1: 1$; analysis of variance showed a marginally significant effect, $F(4,52)=2.35, p<.10$. The trend along the diagonal from top left to lower right of Table 2 is not impressive.

Finally, the set of games tested included a 3 by 3 matrix where one dimension was total game length $(8,16$, and 32$)$ and the other was proportional distance from start or goal, $1 / 4,1 / 2$, and $3 / 4$ of distance from goal, or $3 / 4,1 / 2$, and $1 / 4$ of distance from start. Analysis of variance of this matrix revealed a marginally significant effect for total game length, $F(2,26)=2.78, p<.10$, with means of $34.9,36.9$, and $37.8 \mathrm{~mm}$, respectively, and a significant effect for proportional distance, $F(2,26)=8.72$, $\mathrm{p}<.05$, with means of $38.8,37.1$, and $33.8 \mathrm{~mm}$, respectively, again supporting the Haner and Brown findings. The interaction was not significant, $F(4,52)=1.77, p>.10$.

Haner \& Brown (1955), in their report of response strength subsequent to blocking of goal-directed behavior, interpreted their results in terms of a direct relation between "instigation to action" and goal proximity at the point of blocking. However, their use of a task, or game, or constant length necessarily confounded goal proximity, or distance from the goal, with distance from the start. While the present results support the empirical finding of Haner and Brown, analyses involving games of different lengths suggest that, at least under the conditions of the present study, distance from start is a more potent factor than distance from goal. Also, while the results of this study are not unequivocal, they point to the importance of response-related components in the motivational analysis of goal-directed behavior. Most likely, future research will reveal that the relative contribution of each of these factors, distance from start and goal proximity, depends on special conditions. For example, it is possible that the design of the present experiment, e.g., introducing the situation to $\mathrm{S}$ as a series of games, enhanced the value of instrumental components and diminished the significance of the goal, or reward. Presentation as a working situation with a more valuable reward might shift the balance in favor of goal proximity as the more important factor. REFERENCES

AMSEL, A., ERNHART, C. B., \& GALBRECHT,
C. R. Magnitude of frustration effect and strength of antedating goal factors. Psychological Reports, 1961, 8. 183-186.

HANER. C. F.. \& BROWN, P. A. Clarification of the instigation to action concept in the frustration-aggression hypothesis. Journal of Abnormal \& Social Psychology, 1955, 51, 204-206.

LEWIS, M. Some nondecremental effects of effort. Journal of Comparative \& Physiological Psychology, 1964, 57, 367-372.

\title{
Initial observations on the learning of connected discourse to complete mastery*
}

\author{
DAVID J. KING \\ State University of New York, Oswego, N.Y. 13126
}

Twenty-five Ss learned a 95-word-long story to complete mastery. All recalls of each S were scored for accuracy of recall by two dependent variables, number of words and number of three-word sequencies. The mean number of trials to successive criteria were computed for both word and sequence measures. The relationship between successive criteria and the learning of words is curvilinear, while the relationship between successive criteria and sequence learning is a straight line.

A general procedure that has been developed for studying the retention of connected discourse has involved the intercorrelation of assorted dependent-variable measures. The resultant correlation matrices were then subjected to factor analysis (King, 1960). Factored matrices have ranged from 7 by 7 to 13 by 13 in size. Considering all studies that have been done using this technique (King, 1961, 1966, 1968; King \& Harper, 1967; King \& Madill, 1968; King \& Russell, 1966; King \& Schultz, 1960; King \& Yu, 1962), approximately 100 matrices have been factor analyzed. All but seven of the factor analyses have resolved to a two-factor solution. One factor consistently had high loadings on such variables as number of words, number of letters, number of sentences, etc. This factor has been labeled a quantitative dimension. The second factor had high loadings on idea units and number of sequences found in the recall that also appear in the original. This second factor has been labeled an organizational dimension.

All of the above studies have used recalls of passages collected after a small number (usually once or twice) of presentations.

\footnotetext{
*The author wishes to thank Mr. K. Mack for collecting a portion of the data presented in this study.
}

The present study is an initial effort to determine the usefulness of the two-factor distinction when the learning material is learned to complete mastery. With learning to complete mastery, all variability disappears and the correlational/factoranalytic approach is not possible. 3 hie present study will observe the acquisition function of the quantitative and organizational factors from initial learning through mastery. Distinctive quantitative and organizational acquisition functions would suggest the usefulness of these two factors in future studies.

\section{LEARNING MATERIAL}

The learning material consisted of a 95-word-long version of Bartlett's famous "War of the Ghosts" story (1932). The passage used read as follows: "Two Indians went down to the river to hunt seals. While they were there a canoe came up and the men in the canoe asked the Indians to join them in a war. One Indian went home, the other joined the men as they went to fight. They arrived at Kallama and the battle began. Many were killed on both sides. Then one of the Indians said, 'Let us go, he has been hit.' He felt well but they said he had been hit. When he returned to his village he fell down and died."

The design was a simple comparison of two of the component processes involved in learning a prose passage to complete mastery. 